\title{
The Psychometric Properties and Factor Structure of the Postpartum Bonding Questionnaire in Japanese Mothers
}

\author{
Hitoshi Kaneko*, Shuji Honjo \\ Center for Developmental Clinical Psychology and Psychiatry, Nagoya University, Nagoya, Japan \\ Email: kaneko@cc.nagoya-u.ac.jp
}

Received 27 April 2014; revised 25 May 2014; accepted 21 June 2014

Copyright (C) 2014 by authors and Scientific Research Publishing Inc.

This work is licensed under the Creative Commons Attribution International License (CC BY).

http://creativecommons.org/licenses/by/4.0/

(c) (i) Open Access

\section{Abstract}

The aim of this study was to examine the psychometric properties of the Postpartum Bonding Questionnaire (PBQ) in Japanese mothers. Additionally, we investigated the association between postpartum bonding and postnatal depression. The participants of this study were 1786 Japanese mothers, recruited during their infants' 3 months check-ups at a public health center. The mothers completed the PBQ and the Edinburgh Postnatal Depression Scale (EPDS). We examined the factor structure of the PBQ. However, we were unable to confirm the original 4 factors. A 1-factor solution was extracted by omitting 9 items that did not have significant loadings onto the single factor. According to the cut-off values of the original $P B Q$, the positive rate was $7.7 \%$. The $P B Q$ score was moderately correlated with the EPDS score. The abridged 16-item Japanese version of the PBQ can be used as a reliable screening tool during check-ups for Japanese mothers. Health practitioners should pay attention to mothers who have bonding disturbances at postpartum.

\section{Keywords}

Postpartum Bonding Questionnaire (PBQ), Postpartum Depression, Edinburgh Postnatal Depression Scale (EPDS), Factor Structure

\section{Introduction}

The development of mother-infant emotional bonding is an extremely crucial process in the postpartum period (Brockington, 2011). Previous studies have found that some mothers do not immediately feel affection for their babies after their delivery; indeed, approximately 40\% of primiparae feel indifferent about their newborn infants

${ }^{*}$ Corresponding author. 
when holding their infants for the first time (Robson \& Kumar, 1980). Furthermore, 3\% of mothers express no pleasure in interacting with their infants (Righetti-Veltema, Conne-Perreard, Bousquet, \& Manzano, 2002). When mother-infant interaction is impaired, it adversely affects infants' development (Pearson et al., 2011).

Postpartum depression is generally defined as depression occurring within the first year of childbirth. The prevalence of postpartum depression ranges from 10\% to 15\% (Beck, 2001; Figueiredo \& Conde, 2011; Mann, Gilbody, \& Adamson, 2010). Numerous studies have documented that postpartum depression affects mothers' ability to parent well (Murray, Cooper, \& Hipwell, 2003). In contrast, little attention has been paid to motherinfant emotional bonding at postpartum. There is, however, some research on impairments in mother-infant bonding: for instance, of mothers referred to maternal care units in the UK and New Zealand, $11 \%$ were found to reject their infants (Brockington, Aucamp, \& Fraser, 2006).

The Postpartum Bonding Questionnaire (PBQ) is one of the most commonly used questionnaires for assessing mothers' emotional involvement with their infants during the postpartum period (Brockington, Fraser, \& Wilson, 2006; Brockington, Oates, George, Turner, Vostanis, Sullivan et al., 2001), although there are other, similar questionnaires (Taylor, Atkins, Kumar, Adams, \& Glover, 2005; van Bussel, Spitz, \& Demyttenaere, 2010; Yoshida, Yamashita, Conroy, Marks, \& Kumar, 2012). The PBQ has been widely employed in research and clinical practice (Dearman, Musonda, Roberts, Bowles, \& Morris, 2012; Edhborg, Matthiesen, Lundh, \& Widstrom, 2005; Edhborg, Nasreen, \& Kabir, 2011; Hornstein et al., 2006; Pearson, Lightman, \& Evans, 2011; Reck et al., 2006; Wittkowski, Harvey, \& Wieck, 2007). The PBQ consists of 25 items in 4 subscales: “impaired bonding” (12 items), "rejection and anger" (7 items), "anxiety about care" (4 items), and "risk of abuse" (2 items) (Brockington et al., 2001). Previous research has examined the utility of the PBQ for depressed and psychotic patients in psychiatric units (Chandra, Bhargavaraman, Raghunandan, \& Shaligram, 2006; Moehler, Brunner, Wiebel, Reck, \& Resch, 2006; Noorlander, Bergink, \& van den Berg, 2008). As for its utility specifically in Asian countries, Siu, Ip, Chow, Kwok, Li, Koo et al. (2010) validated the Chinese version of the PBQ in a population of mothers in Hong Kong; they conducted structured interviews and administered the Chinese version of the PBQ to depressed patients and found that the scale had a sensitivity of $83 \%$ and a specificity of $96 \%$.

Although much research on mother infant bonding has focused on clinical samples, little has focused on mother-infant bonding in the general population. In Japan, all local governments organize health check-ups for 18-month-old infants and 36-month-old toddlers, according to the Maternal and Child Health Act (Act No. 141 of 1965). In addition, most local governments have added check-ups for 3-month-old infants as well. All such health check-ups are completely free of charge; as such, approximately $95 \%$ of mothers and children undergo them. These check-ups would be a suitable opportunity to screen for mothers who are having problems in bonding with their infants.

However, with regard to the factor solution of the PBQ, previous studies have presented inconsistent results. The most recent study revealed a 3-factor solution (Wittkowski, Williams, \& Wieck, 2010), but an earlier study reported a 1-factor solution (Reck et al., 2006). Thus, these conflicting results must be resolved through further research.

The aim of this study was to examine the psychometric properties of the PBQ in a large sample of Japanese mothers. Additionally, we aimed to investigate the association between postpartum bonding and postnatal depression in Japanese mothers.

\section{Method}

\subsection{Participants}

The participants of this study were 1786 Japanese mothers, recruited at their infants' 3-month check-ups at the public health center in Hekinan City, Aichi Prefecture, Japan, between May 2008 and January 2011. Hekinan City has a population of approximately 74,000, making it a medium-sized city by Japan standards. Seven hundred and ninety babies were born in 2008.

The mothers' ages ranged from 16 to 45 years $(M=29.8$ years, $S D=5.0)$. Half of the infants were male (51.7\%), their mean age was 123.6 days ( $\mathrm{SD}=9.6)$, their mean gestational age was 38.9 weeks $(\mathrm{SD}=1.5)$, and their mean weight at birth was $3009.8 \mathrm{~g}(\mathrm{SD}=407.3)$. Approximately half of mothers $(45.0 \%)$ were primiparas. Forty-nine percent of the mothers were employed before this pregnancy. Additionally, $1.2 \%$ were not married or had no partner. Furthermore, 58.6\% were breastfeeding their babies, $17.2 \%$ were both bottle-feeding and breastfeeding, and $23.8 \%$ were only bottle-feeding. 
The mothers were asked to complete the questionnaires, which were sent to them by mail, about their infants' and their own health conditions. These questionnaires were then collected at the infants' 3-month check-ups at the public health center. The exclusion criteria were as follows: 1) being unable to read Japanese and 2) being multiparous. The study protocol was approved by the institutional ethical review board of the Graduate School of Education and Human Development, Nagoya University.

\subsection{Instruments}

Postpartum Bonding Questionnaires. The PBQ (Brockington et al., 2001) is a 25-item scale assessing mothers' attitudes towards their infants. Each item is rated on a 6-point Likert scale ranging from 0 (always) to 5 (never). The PBQ was first translated into Japanese by bilingual Japanese-English speakers, and then back-translated into English. All of the back-translated items were confirmed by the original author.

Edinburgh Postnatal Depression Scale (EPDS). The Edinburgh Postnatal Depression Scale (EPDS) was employed (Cox, Holden, \& Sagovsky, 1987) to assess participants' postnatal depression levels. There are a total of 10 items on the EPDS, and the maximum total score is 30. The Japanese version of the EPDS was validated (Okano et al., 1996). In Western populations, a score of 13 and above is regarded as indicative of postnatal depression (Cox et al., 1987); however, among Japanese women, a score of 9 and above has been established as the cut-off point (Okano et al., 1996; Yamashita, Yoshida, Nakano, \& Tashiro, 2000). The sensitivity of the Japanese version of the EPDS is $82 \%$, and its specificity is $95 \%$ for women in the postpartum period (Yamashita et al., 2000).

\subsection{Statistical Analysis}

The factor structure of the PBQ was examined using factor analysis with unweighted leaset-squares method. In order to accurately determine the number of factors, we calculated the eigenvalues for each factor and examined a scree plot. Descriptive statistics are presented as means and standard deviations. Cronbach's alpha coefficients were calculated to determine internal consistency. Finally, we used Pearson's correlation coefficient to assess the association between postpartum bonding and depression. All statistical analyses were conducted using SPSS 17.0.

\section{Results}

\subsection{Factor Analysis}

First, we examined the original 4-factor solution of the PBQ, which accounted for $44.6 \%$ of the total variance. Factor 1 had an eigenvalue of 5.97 and accounted for $23.9 \%$ of the total variance. Factor 2 (eigenvalue $=2.34$ ) accounted for $9.3 \%$ of the variance, factor 3 (eigenvalue $=1.66$ ) for $6.6 \%$, and factor 4 (eigenvalue $=1.20$ ) accounted for $4.8 \%$. However, the difference in eigenvalues between factor 4 and 5 (eigenvalue =1.15) was small; therefore, the original 4-factor solution was not supported (Figure 1).

Thus, we investigated another factor solution of the PBQ. Although 6 factors had eigenvalues $>1$, we observed a sharp drop after the first factor. Therefore, we extracted a 1-factor solution. This factor accounted for 23.9\% of the total variance. Table 1 shows the factor loadings for each item. Loadings above .40 were considered, meaning that 9 items $(3,4,6,9,17,18,20,23,24)$ did not have high enough loadings on the single factor. After omitting these 9 items, we conducted another factor analysis on the 16 remaining items that had loadings above .40. Factor 1 had an eigenvalue of 5.02, which accounted for 31.4\% of the total variance. Further analyses were conducted using both the 16-item and 25-item Japanese versions.

\subsection{Descriptive Statistics}

The mean score on the 16-item version of the Japanese PBQ was $9.3(\mathrm{SD}=7.0)$, while the mean score on the 25-item version was $10.8(\mathrm{SD}=8.3)$. The mean scores for the 4 subscales of the 25 -item version were as follows: impaired bonding, $5.0(\mathrm{SD}=4.4)$; rejection and anger, $2.0(\mathrm{SD}=2.6)$; anxiety and care, $3.7(\mathrm{SD}=2.7)$; and risk of abuse, $0.05(\mathrm{SD}=0.3)$.

According to the previously reported cut-off values (Brockington, Fraser et al. 2006), the positive rate for the total 25 -item scale was $5.3 \%$. The positive rates of the subscales were as follows: impaired bonding, $7.7 \%$; rejection and anger, $0.4 \%$; anxiety and care, $2.2 \%$; and risk of abuse, $0.08 \%$. 


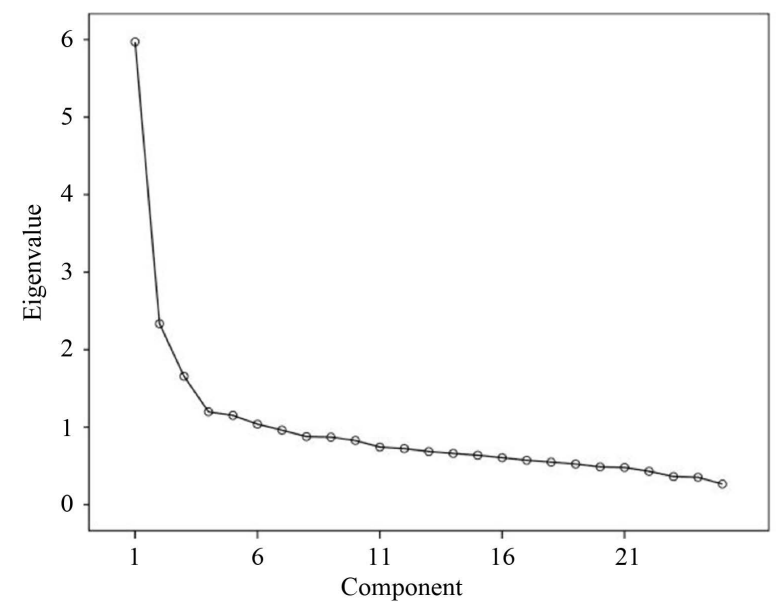

Figure 1. Scree plot for factor analysiys of the PBQ.

Table 1. Loadings and communalities of postpartum bonding questionnaire.

\begin{tabular}{|c|c|c|c|}
\hline No. & Items & Loadings & Communalities \\
\hline 14 & I feel angry with my baby & .686 & .471 \\
\hline 7 & My baby winds me up & .660 & .436 \\
\hline 10 & My baby irritates me & .635 & .403 \\
\hline 13 & I feel trapped as a mother & .562 & .316 \\
\hline 11 & I enjoy playing with my baby & -.549 & .301 \\
\hline 21 & My baby annoys me & .519 & .269 \\
\hline 8 & I love my baby to bits & -.516 & .266 \\
\hline 19 & My baby makes me feel anxious & .500 & .250 \\
\hline 2 & I wish the old days when I had no baby would come back & .499 & .249 \\
\hline 12 & My baby cries too much & .448 & .201 \\
\hline 5 & I regret having this baby & .443 & .196 \\
\hline 22 & I feel confident when caring for my baby & -.420 & .177 \\
\hline 1 & I feel close to my baby & -.418 & .175 \\
\hline 16 & My baby is the most beautiful baby in the world & -.418 & .175 \\
\hline 15 & I resent my baby & .418 & .174 \\
\hline 25 & My baby is easily comforted & -.411 & .169 \\
\hline 24 & I feel like hurting my baby & .397 & .158 \\
\hline 23 & I feel the only solution is for someone else to look after my baby & .357 & .127 \\
\hline 3 & I feel distant from my baby & .347 & .120 \\
\hline 17 & I wish my baby would somehow go away & .329 & .108 \\
\hline 9 & I feel happy when my baby smiles or laughs & -.326 & .106 \\
\hline 18 & I have done harmful things to my baby & .325 & .106 \\
\hline 4 & I love to suddle my baby & -.323 & .104 \\
\hline 20 & I am afraid of my baby & .315 & .099 \\
\hline 6 & The baby does not seem to be mine & .272 & .074 \\
\hline
\end{tabular}




\subsection{Reliability}

Cronbach's alpha coefficient of the 16-item Japanese version was .85; Cronbach's alpha coefficient for the 25-item version was .86. Broken down by subscale, the Cronbach's alpha coefficients were as follows: impaired bonding, .77; rejection and anger, .65; anxiety and care, .55; and risk of abuse, .61.

\subsection{Association between Postpartum Bonding and Depression}

Participants' mean score on the EPDS was 2.83 (SD = 3.46). The EPDS positive rate, when using a cut-off point of greater than or equal to 9 , was $7.1 \%$.

The correlation coefficient between the 25-item PBQ total score and the EPDS was .47 $(p<.001)$, while that between the 16-item version and the EPDS was roughly similar $(r=.46, p<.001)$.

\section{Discussion}

\subsection{Factor Structure and Psychometric Properties of the PBQ}

The present study was the first investigation on the factor structure of the Japanese version of the PBQ using a large sample of Japanese mothers. We did not extract the original 4-factor solution of the PBQ; our results instead suggest that the PBQ has a 1-factor solution in Japanese mothers. Although we ended up excluding 9 items from the original 25-item PBQ with low loadings onto the single factor, the reliability was good, and remained similar to the total 25-item version. We suggest that the abridged questionnaire would be useful for screening mothers during their infants' regular check-ups, as it would be less demanding for both mothers and health practitioners. There are discrepancies with the factor structure of the PBQ. Our findings suggest that a 1-factor solution is suitable for the Japanese general population, which supports a study performed in Heidelberg, Germany (Reck et al., 2006). However, another study reported a 3-factor solution (Wittkowski et al., 2010). A plausible explanation for these differing results could be the differences in the samples. Wittkowski et al. (2010) investigated mothers who were psychiatric inpatients, whereas Reck et al. (2006) investigated mothers from the general population. Taking our results and those of previous studies into consideration, a 1-factor solution appears to be suitable for screening the mothers from the general population.

The positive rate of the impaired bonding subscale in the present study was $7.7 \%$, when using the original cutoff points. This rate is similar to that found in a previous study on a general population sample in Heidelberg (7.1\%; Reck et al., 2006). Regarding the other subscales, the positive rates were consistent with those reported in the Heidelberg study, although there were differences in the samples and survey periods: the current study surveyed mothers 3 months postpartum, while Reck et al. (2006) surveyed mothers 2 weeks postpartum. This high positive rate in the general population-which indicates that approximately $7 \%$ of mothers might have bonding disturbances postpartum—-suggests that health practitioners should pay close attention when screening mothers.

Nevertheless, researchers must be aware of the possible influence of response bias due to social desirability (Reck et al., 2006). Previous studies found that total PBQ score had small but significant correlations with scores on a measure of social desirability at 20-25 weeks postpartum $(r=.27$; van Bussel et al., 2010). In the current study, we collected the data during infants' 3-month check-ups at a public health center using a method by which mothers would be identified. Therefore, mothers might be concerned about revealing their impaired bonding to public health center staff because they feel ashamed of it. However, several studies found that there were no differences in depressive symptomatology (Nishizono-Maher et al., 2004) or difficulty in coping with infants (Matthey, White, \& Rice, 2010) between mothers who were identifiable when surveyed and those who were surveyed anonymously. Thus, it should be interpreted cautiously whether social desirability has an effect on PBQ score.

\subsection{EPDS Scores}

Our findings revealed that the EPDS positive rate was $7.3 \%$, which is lower than that previously reported in Western countries. This can be explained by the effect of cultural factors on EPDS positive rates (Halbreich \& Karkun, 2006). Furthermore, there are discrepancies in EPDS positive rates at 3-4 months postpartum in Japanese mothers, ranging from 6.7\% (Tamaki, Murata, \& Okano, 1997) to 13.9\% (Nishizono-Maher et al., 2004).

One possible explanation for the different results in Japan could be differences in sampling locations. In met- 
ropolitan areas, the EPDS positive rate is approximately 13\% (Nishizono-Maher et al., 2004; Watanabe et al., 2008); however, in a different medium-sized city from that sampled in this study, the EPDS positive rate at 4 months postpartum was $6.7 \%$ (Tamaki et al., 1997). Our findings are in accord with the results reported by Tamaki et al. (1997). In Japanese medium-size cities, more mothers can take up residence in areas close to their original home or their husband's original home compared with mothers living in metropolitan areas, due to the comparatively inexpensive rental fees and land prices. Therefore, mothers in the present study might have received social support more easily from their own mothers or husband's mothers than mothers who live in metropolitan areas. In addition, previous studies found that social support from family members relates to mothers' mental health (Arimoto \& Murashima, 2007). Further research is needed to elucidate the precise factors that influence the positive EPDS rate among Japanese mothers.

\subsection{Relationship between Postpartum Bonding and Depression}

Our findings revealed moderate correlations between PBQ scores and EPDS scores. These findings are comparable with those of previous studies ( $r=.43$, Reck et al., 2006; $r=.41$, van Bussel et al. 2010). Furthermore, a recent Japanese study also reported correlations between maternal bonding and depression at 1 month $(r=.46$, Kokubu, Okano, \& Sugiyama, 2012) and at 4 months postpartum ( $r=.39$, Yoshida et al., 2012), although they used the Mother Infant Bonding Scale (Kumar, 1997; Taylor et al., 2005), instead of the PBQ. Another study reported strong correlations between postpartum bonding and maternal depression $(r=.71)$, however, the authors investigated inpatient mothers admitted to psychiatric wards and used the Beck Depression Inventory for assessing depression (Wittkowski et al., 2010).

Our findings suggest that approximately $16 \%$ of the variance accounted for the correlations between the PBQ and the EPDS, meaning that some mothers had low depression scores but high impaired bonding scores. In addition, previous studies addressed the importance of distinguishing between bonding disturbance and postpartum depression (Brockington, 1996). Taking that into consideration, we suggest using inventories that assess both depression and bonding when screening mothers from the general population.

\subsection{Limitations}

The present study has several limitations. First, the Japanese version of the PBQ does not have validated cut-off values. In order for this scale to be effective in screening for postpartum bonding disorder, future research should conduct diagnostic interviews on participants to help develop cut-off values for the Japanese version of the PBQ. Second, we did not use a structured clinical interview. Thus, a diagnosis of depression could not be made in this study.

Taking these shortcomings into consideration, the strength of the present study was that it examined the psychometric properties of the PBQ in a large sample of Japanese mothers. Furthermore, we were able to create an abridged 16-item Japanese version of the PBQ that can be implemented as a screening tool during regular checkups for Japanese mothers.

\section{Summary}

The present study showed that Japanese version of the PBQ has a 1-factor solution in a Japanese community sample, whereas the original PBQ has a 4-factor solution. Thus, the Japanese version of the PBQ, consisting of only 16 items, can be considered an abridged version. When assessing postnatal mothers with mental health problems, it is important to include information about mother-infant bonding difficulties.

\section{Acknowledgements}

This study was supported by Grants-in-Aid for Yong Scientists (B) from the Ministry of Education, Culture, Sports Science and Technology of Japan (19730428 and 21730547). The authors thank the staff members at Hekinan City Public Health Center.

\section{References}

Arimoto, A., \& Murashima, S. (2007). Child-Rearing Anxiety and Its Correlates among Japanese Mothers Screened at 18-Month Infant Health Checkups. Public Health Nursing, 24, 101-110. 
http://dx.doi.org/10.1111/j.1525-1446.2007.00614.x

Beck, C. T. (2001). Predictors of Postpartum Depression: An Update. Nursing Research, 50, 275-285. http://dx.doi.org/10.1097/00006199-200109000-00004

Brockington, I. (1996). Motherhood and mental health. Oxford: Oxford University Press.

Brockington, I. (2011). Maternal Rejection of the Young Child: Present Status of the Clinical Syndrome. Psychopathology, 44, 329-336. http://dx.doi.org/10.1159/000325058

Brockington, I. F., Aucamp, H. M., \& Fraser, C. (2006). Severe Disorders of the Mother-Infant Relationship: Definitions and Frequency. Archives of Women's Mental Health, 9, 243-251. http://dx.doi.org/10.1007/s00737-006-0133-0

Brockington, I. F., Fraser, C., \& Wilson, D. (2006). The Postpartum Bonding Questionnaire: A Validation. Archives of Women's Mental Health, 9, 233-242. http://dx.doi.org/10.1007/s00737-006-0132-1

Brockington, I. F., Oates, J., George, S., Turner, D., Vostanis, P., Sullivan, M. et al. (2001). A Screening Questionnaire for Mother-Infant Bonding Disorders. Archives of Women's Mental Health, 3, 133-140.

http://dx.doi.org/10.1007/s007370170010

Chandra, P. S., Bhargavaraman, R. P., Raghunandan, V., \& Shaligram, D. (2006). Delusions Related to Infant and Their Association with Mother-Infant Interactions in Postpartum Psychotic Disorders. Archives of Women's Mental Health, 9, 285-288. http://dx.doi.org/10.1007/s00737-006-0147-7

Cox, J. L., Holden, J. M., \& Sagovsky, R. (1987). Detection of Postnatal Depression: Development of the 10-Item Edinburgh Postnatal Depression Scale. British Journal of Psychiatry, 150, 782-786. http://dx.doi.org/10.1192/bjp.150.6.782

Dearman, L., Musonda, P., Roberts, F., Bowles, K., \& Morris, E. (2012). Bonding in Women with Postnatal Anaemia: A Pilot Case Control Study Looking at Postnatal Bonding in Women Who Have Been Diagnosed with Anaemia at a University Hospital in the East of England. Archives of Gynecology and Obstetrics, 285, 1243-1248.

http://dx.doi.org/10.1007/s00404-011-2142-0

Edhborg, M., Matthiesen, A. S., Lundh, W., \& Widstrom, A. M. (2005). Some Early Indicators for Depressive Symptoms and Bonding 2 Months Postpartum-A Study of New Mothers and Fathers. Archives of Women's Mental Health, 8, 221-231. http://dx.doi.org/10.1007/s00737-005-0097-5

Edhborg, M., Nasreen, H. E., \& Kabir, Z. N. (2011). Impact of Postpartum Depressive and Anxiety Symptoms on Mothers' Emotional Tie to Their Infants 2-3 Months Postpartum: A Population-Based Study from Rural Bangladesh. Archives of Women's Mental Health, 14, 307-316. http://dx.doi.org/10.1007/S00737-011-0221-7

Figueiredo, B., \& Conde, A. (2011). Anxiety and Depression in Women and Men from Early Pregnancy to 3-Months Postpartum. Archives of Women's Mental Health, 14, 247-255. http://dx.doi.org/10.1007/S00737-011-0217-3

Halbreich, U., \& Karkun, S. (2006). Cross-Cultural and Social Diversity of Prevalence of Postpartum Depression and Depressive Symptoms. Journal of Affective Disorders, 91, 97-111. http://dx.doi.org/10.1016/j.jad.2005.12.051

Hornstein, C., Trautmann-Villalba, P., Hohm, E., Rave, E., Wortmann-Fleischer, S., \& Schwarz, M. (2006). Maternal Bond and Mother-Child Interaction in Severe Postpartum Psychiatric Disorders: Is There a Link? Archives of Women's Mental Health, 9, 279-284. http://dx.doi.org/10.1007/s00737-006-0148-6

Kokubu, M., Okano, T., \& Sugiyama, T. (2012). Postnatal Depression, Maternal Bonding Failure, and Negative Attitudes towards Pregnancy: A Longitudinal Study of Pregnant Women in Japan. Archives of Women's Mental Health, 15, 211216. http://dx.doi.org/10.1007/s00737-012-0279-x

Kumar, R. C. (1997). “Anybody’s Child”: Severe Disorders of Mother-to-Infant Bonding. British Journal of Psychiatry, 171, 175-181. http://dx.doi.org/10.1192/bjp.171.2.175

Mann, R., Gilbody, S., \& Adamson, J. (2010). Prevalence and Incidence of Postnatal Depression: What Can Systematic Reviews Tell Us? Archives of Women's Mental Health, 13, 295-305. http://dx.doi.org/10.1007/s00737-010-0162-6

Matthey, S., White, T., \& Rice, S. (2010). Women’s Responses to Postnatal Self-Report Mood and Experience Measures: Does Anonymity Make a Difference? Archives of Women's Mental Health, 13, 477-484. http://dx.doi.org/10.1007/s00737-010-0159-1

Moehler, E., Brunner, R., Wiebel, A., Reck, C., \& Resch, F. (2006). Maternal Depressive Symptoms in the Postnatal Period are Associated with Long-Term Impairment of Mother-Child Bonding. Archives of Women's Mental Health, 9, 273-278. http://dx.doi.org/10.1007/s00737-003-0007-7

Murray, L., Cooper, P., \& Hipwell, A. (2003). Mental Health of Parents Caring for Infants. Archives of Women's Mental Health, 6, S71-S77. http://dx.doi.org/10.1007/s00737-003-0007-7

Nishizono-Maher, A., Kishimoto, J., Yoshida, H., Urayama, K., Miyato, M., Otsuka, Y., \& Matsui, H. (2004). The Role of Self-Report Questionnaire in the Screening of Postnatal Depression-A Community Sample Survey in Central Tokyo. Social Psychiatry and Psychiatric Epidemiology, 39, 185-190. http://dx.doi.org/10.1007/s00127-004-0727-7

Noorlander, Y., Bergink, V., \& van den Berg, M. P. (2008). Perceived and Observed Mother-Child Interaction at Time of 
Hospitalization and Release in Postpartum Depression and Psychosis. Archives of Women's Mental Health, 11, 49-56. http://dx.doi.org/10.1007/s00737-008-0217-0

Okano, T., Murata, M., Masuji, F., Tamaki, R., Nomura, J., Miyaoka, H., \& Kitamura, T. (1996). Validation and Reliability of Japanese Version of EPDS (Edinburgh Postnatal Depression Scale). Archives of Psychiatric Diagnostics and Clinical Evaluation, 7, 525-533 (in Japanese).

Pearson, R., Lightman, S., \& Evans, J. (2011). Attentional Processing of Infant Emotion during Late Pregnancy and Mother-Infant Relations after Birth. Archives of Women's Mental Health, 14, 23-31. http://dx.doi.org/10.1007/s00737-010-0180-4

Pearson, R. M., Heron, J., Melotti, R., Joinson, C., Stein, A., Ramchandani, P. G., \& Evans, J. (2011). The Association between Observed Non-Verbal Maternal Responses at 12 Months and Later Infant Development at 18 Months and IQ at 4 Years: A Longitudinal Study. Infant Behavior \& Development, 34, 525-533. http://dx.doi.org/10.1016/J.Infbeh.2011.07.003

Reck, C., Klier, C. M., Pabst, K., Stehle, E., Steffenelli, U., Struben, K., \& Backenstrass, M. (2006). The German Version of the Postpartum Bonding Instrument: Psychometric Properties and Association with Postpartum Depression. Archives of Women's Mental Health, 9, 265-271.http://dx.doi.org/10.1007/s00737-006-0144-x

Righetti-Veltema, M., Conne-Perreard, E., Bousquet, A., \& Manzano, J. (2002). Postpartum Depression and Mother-Infant Relationship at 3 Months old. Journal of Affective Disorders, 70, 291-306. http://dx.doi.org/10.1016/S0165-0327(01)00367-6

Robson, K. M., \& Kumar, R. (1980). Delayed Onset of Maternal Affection after Childbirth. British Journal of Psychiatry, 136, 347-353. http://dx.doi.org/10.1192/bjp.136.4.347

Siu, B. W. M., Ip, P., Chow, H. M. T., Kwok, S. S. P., Li, O. L., Koo, M. L. et al. (2010). Impairment of Mother-Infant Relationship Validation of the Chinese Version of Postpartum Bonding Questionnaire. Journal of Nervous and Mental Disease, 198, 174-179. http://dx.doi.org/10.1097/NMD.0b013e3181d14154

Tamaki, R., Murata, M., \& Okano, T. (1997). Risk Factors for Postpartum Depression in Japan. Psychiatry and Clinical Neurosciences, 51, 93-98. http://dx.doi.org/10.1111/j.1440-1819.1997.tb02368.x

Taylor, A., Atkins, R., Kumar, R., Adams, D., \& Glover, V. (2005). A New Mother-to-Infant Bonding Scale: Links with Early Maternal Mood. Archives of Women's Mental Health, 8, 45-51.

van Bussel, J. C., Spitz, B., \&Demyttenaere, K. (2010). Three Self-Report Questionnaires of the Early Mother-to-Infant Bond: Reliability and Validity of the Dutch Version of the MPAS, PBQ and MIBS. Archives of Women's Mental Health, 373-384. http://dx.doi.org/10.1007/s00737-009-0140-Z

Watanabe, M., Wada, K., Sakata, Y., Aratake, Y., Kato, N., Ohta, H., \& Tanaka, K. (2008). Maternity Blues as Predictor of Postpartum Depression: A Prospective Cohort Study among Japanese Women. Journal of Psychosomatic Obstetrics and Gynecology, 29, 206-212. http://dx.doi.org/10.1080/01674820801990577

Wittkowski, A., Harvey, J., \& Wieck, A. (2007). A Survey of Instruments Used for the Psychological Assessment of Patients Admitted to Mother and Baby Inpatient Facilities. Archives of Women's Mental Health, 10, 237-240. http://dx.doi.org/10.1007/s00737-007-0195-7

Wittkowski, A., Williams, J., \& Wieck, A. (2010). An Examination of the Psychometric Properties and Factor Structure of the Post-Partum Bonding Questionnaire in a Clinical Inpatient Sample. British Journal of Clinical Psychology, 49, 163172. http://dx.doi.org/10.1348/014466509x445589

Yamashita, H., Yoshida, K., Nakano, H., \& Tashiro, N. (2000). Postnatal Depression in Japanese Women: Detecting the Early Onset of Postnatal Depression by Closely Monitoring the Postpartum Mood. Journal of Affective Disorders, 58, 145-154. http://dx.doi.org/10.1016/S0165-0327(99)00108-1

Yoshida, K., Yamashita, H., Conroy, S., Marks, M., \& Kumar, C. (2012). A Japanese Version of Mother-to-Infant Bonding Scale: Factor Structure, Longitudinal Changes and Links with Maternal Mood during the Early Postnatal Period in Japanese mothers. Archives of Women's Mental Health, 15, 343-352. http://dx.doi.org/10.1007/s00737-012-0291-1 
Scientific Research Publishing (SCIRP) is one of the largest Open Access journal publishers. It is currently publishing more than 200 open access, online, peer-reviewed journals covering a wide range of academic disciplines. SCIRP serves the worldwide academic communities and contributes to the progress and application of science with its publication.

Other selected journals from SCIRP are listed as below. Submit your manuscript to us via either submit@scirp.org or Online Submission Portal.
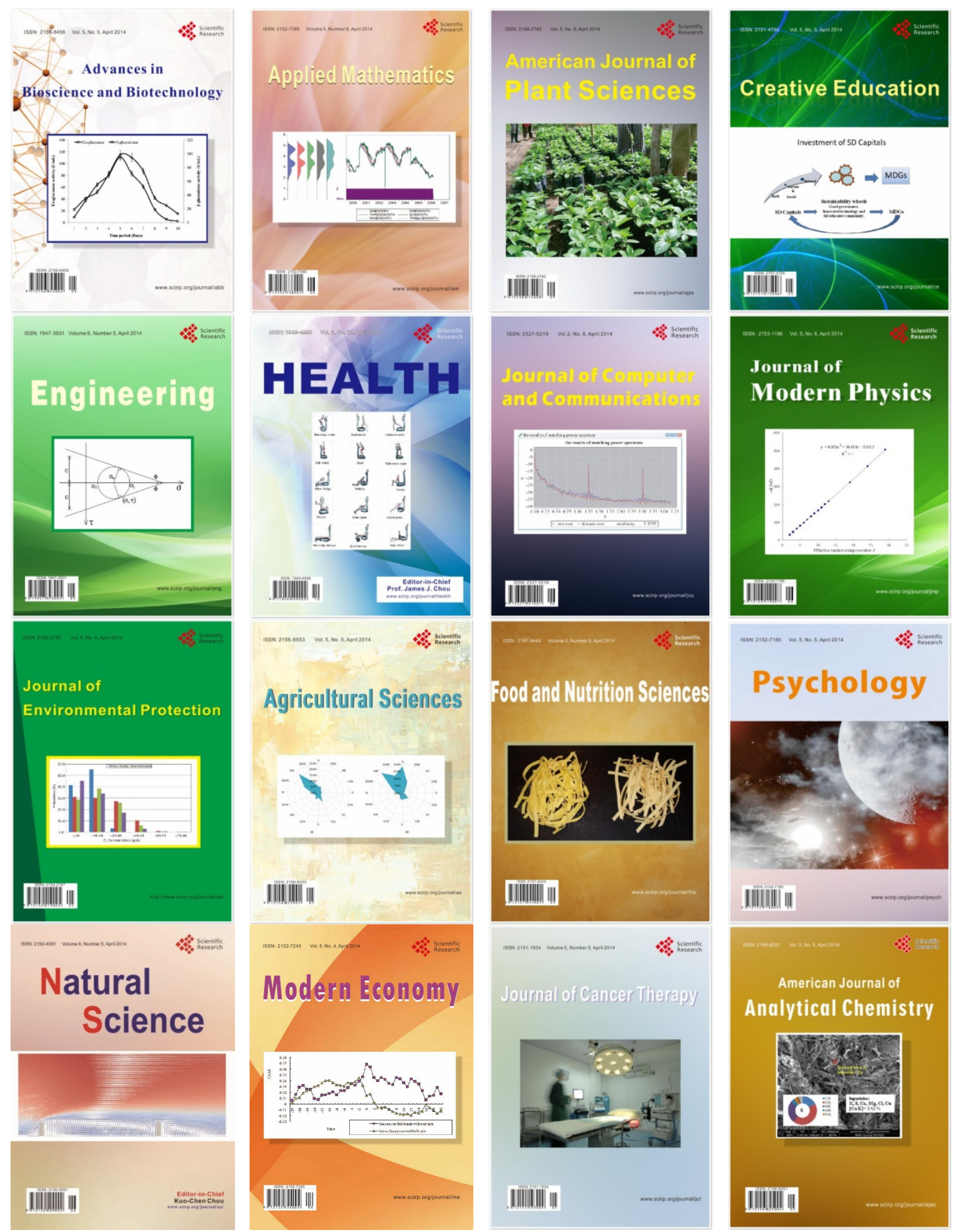